\title{
PERSPECTIVE Psychiatric blood biomarkers: avoiding jumping to premature negative or positive conclusions
}

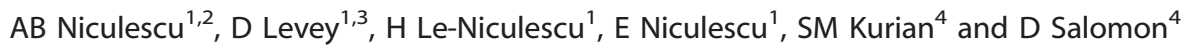

Blood biomarkers may provide a scientifically useful and clinically usable peripheral signal in psychiatry, as they have been doing for other fields of medicine. Jumping to premature conclusions, negative or positive, can create confusion in this field. Reproducibility is a hallmark of good science. We discuss some recent examples from this dynamic field, and show some new data in support of previously published biomarkers for suicidality (SAT1, MARCKS and SKA2). Methodological clarity and rigor in terms of biomarker discovery, validation and testing is needed. We propose a set of principles for what constitutes a good biomarker, similar in spirit to the Koch postulates used at the birth of the field of infectious diseases.

Molecular Psychiatry (2015) 20, 286-288; doi:10.1038/mp.2014.180; published online 13 January 2015

... 'With all thy getting, get understanding' ... -Proverbs 4:7 King James Version (KJV)

The identification of blood biomarkers for disease risk has emerged as an important area of translational research in medicine, particularly in cancer ${ }^{1}$ and cardiovascular medicine, ${ }^{2}$ in the quest for precision and individualization of preventive measures and of treatment. Although genetic tests may have a useful role as well ${ }^{3}$ and can be done early on in life, biomarkers that look at gene expression, proteomic or metabolomic characteristics better reflect the gene-environment interactions that lead to disease manifestation. In psychiatry, in particular, it is impractical to directly access the target organ - the brain-in live individuals, and even its proxy fluid, the cerebrospinal fluid, is less accessible for routine use than the blood. Although it is clear that the blood is not the brain, there are common biological mechanisms, environmental and medication effects across tissues that can be identified with convergent approaches. $^{4,5}$ Upon demonstration of reproducibility and predictive ability in independent cohorts, the key litmus tests, such biomarkers should be rapidly moved into population testing and validation for clinical use. The unmet need in psychiatry is great, and the potential of biomarkers to revolutionize clinical management is commensurate with that. However, the burden of proof needs to be high as well.

We have recently published a study detailing the identification of blood gene expression biomarkers for suicidality. ${ }^{6}$ The discovery was carried out using a powerful but hard to accomplish longitudinal within-subject design in male bipolar subjects, a high-risk group for suicide, identifying a small but very valuable subgroup of subjects who exhibited major switches in suicidal ideation at the time of different testing visits. Differential gene expression studies within each subject were carried out, factoring out any genetic and a majority of environmental effects. (Self-)report of phenotype is also more accurate in a within-subject design. In the effect, this design is arguably better than an identical twin study. What was changed in expression in common across the different subjects was carried forward in the analysis. A convergent functional genomics (CFG) approach was then used to prioritize the differentially expressed gene list, using independent lines of evidence implicating them in suicide (human genetic studies, human postmortem brain studies). Validation of the genes prioritized by CFG was carried out by testing for changes in expression in the blood of a demographically matched for gender and age cohort of male violent (non-overdose) suicide completers from the coroners' office. Six genes (SAT1, MARCKS, UBA6, PTEN, MAP3K and MT-ND6) showed a statistically significant, Bonferroni corrected, stepwise change in expression from live bipolar subjects in no suicidal states to high suicidal states to suicide completers. To further validate the markers and demonstrate their predictive ability, we examined in a larger male bipolar cohort whether biomarker levels can predict future psychiatric hospitalizations for suicidality, and demonstrated that they did. The panel of six markers had an AUC of 0.73 , and a $P$-value of 0.04 . This work has opened the field to active exploration, and raised hopes for progress. What was not known at the time, and is an area of active exploration by us and others, was: (1) whether these biomarkers work in diagnoses other than bipolar disorder or there are other biomarkers better suited for other diagnoses, (2) whether gender has a role, that is, would a different set of biomarkers work in females and (3) whether type of suicide (violent vs non-violent and impulsive vs planned) is underpinned by different sets of markers. The answer to these questions is likely to be at least partially yes. Gene expression is powerfully modulated by disease biology and medication effects in the first instance, by gender biology and sex hormones in the second instance, and by stress reactivity, drug abuse and toxicology in the third instance. Since the publication of our work in 2013, a study by Weinberger and colleagues ${ }^{7}$ has replicated one of our six markers, MARCKS, in the brains of male violent suicide completers with schizophrenia, but not those with depression or with non-violent suicide. Another study by Kaminsky and colleagues ${ }^{8}$ identified a new biomarker for suicide, SKA2, primarily in depression cohorts. Interestingly, SKA2 has effects upstream of our top biomarkers, SAT1, and may regulate its transcription.

${ }^{1}$ Department of Psychiatry and Medical Neuroscience, Indiana University School of Medicine, Indianapolis, IN, USA; ${ }^{2}$ Indianapolis VA Medical Center, Indianapolis, IN, USA ${ }^{3}$ Program in Medical Neuroscience, Paul and Carole Stark Neurosciences Research Institute, Indiana University School of Medicine, Indianapolis, IN, USA and ${ }^{4}$ Department of Molecular and Experimental Medicine, The Scripps Research Institute, La Jolla, CA, USA. Correspondence: Professor AB Niculescu, Department of Psychiatry, Indiana University School of Medicine, Neuroscience Research Building, Room 200B, 320 West 15th Street, Indianapolis, IN 46202, USA.

E-mail: anicules@iupui.edu

Received 22 October 2014; revised 12 November 2014; accepted 14 November 2014; published online 13 January 2015 
SAT1 (203455_s_at)

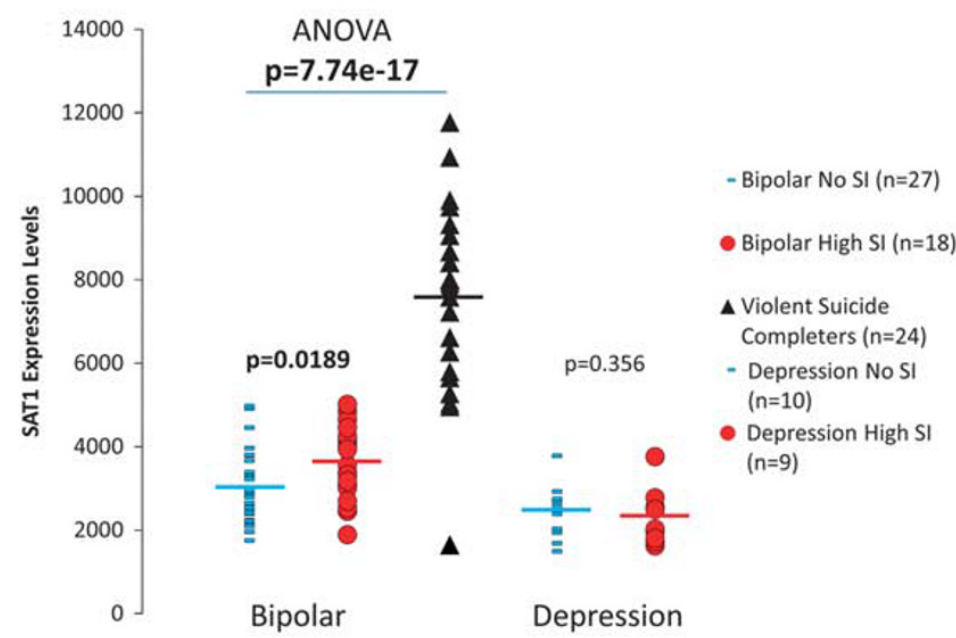

MARCKS (213002_at)

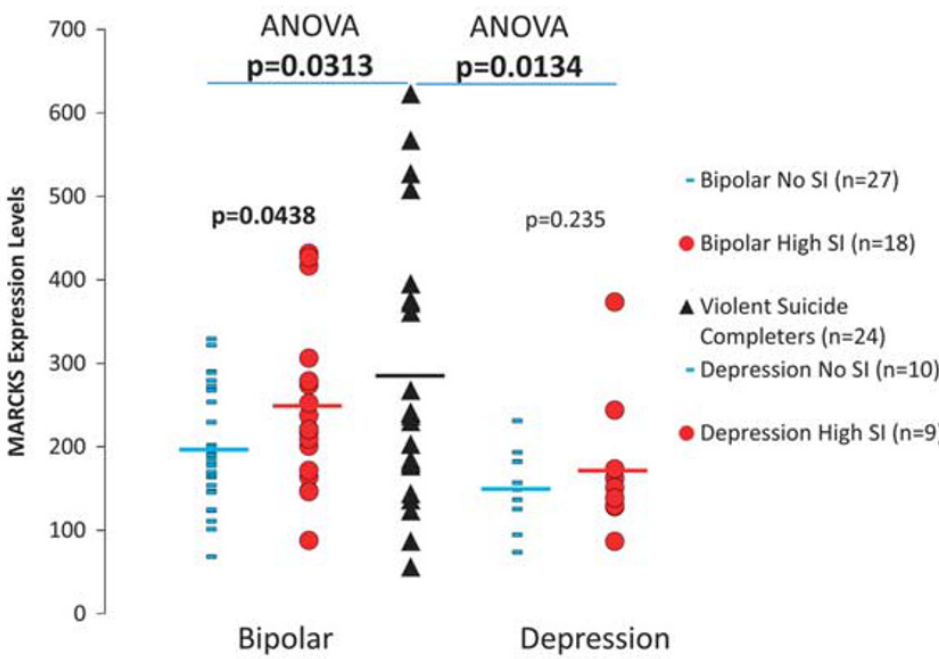

SKA2(225686_at)

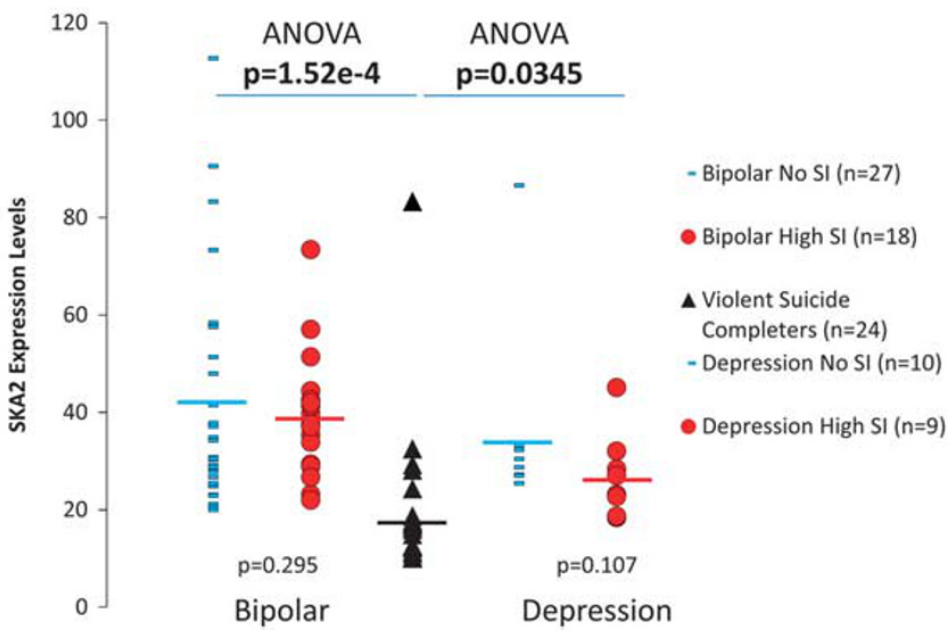

Figure 1. Reproducibility and diagnostic differences for top biomarkers for suicidality. Methods are as previously described. ${ }^{6}$ Demographics of cohorts are presented in Supplementary Table S1. SAT1 and MARCKS, previously identified as top blood biomarkers increased in expression in suicidality by our group, ${ }^{6}$ are significantly increased in violent suicide completers and in live subjects with high suicidal ideation (SI) in bipolar disorder, but not depression. SKA2, previously identified as a blood biomarker decreased in expression in suicidality by Kaminsky and colleagues, ${ }^{8}$ is significantly decreased in violent suicide completers. 
A study just reported by Mullins et al. ${ }^{9}$ purports not to replicate our six top biomarkers. The authors jump to the conclusion of wanting to make a general statement in that regard. However, the study was carried out: (1) in depression as opposed to our bipolar work, (2) in a cohort that was $75 \%$ female as opposed to our exclusively male cohorts and (3) in a different phenotype/type of suicidality (treatment emergent suicidal ideation in response to antidepressant treatment). Moreover, the probands and controls were not matched for age ( $P$-value for age difference was significant at $P=0.02)$. That may not matter much in the genetic (DNA) research arena in which the senior study authors have a good prior track record and expertise. For gene expression (RNA), however, age matters. Particularly unfortunate, given the fact that their sample was $75 \%$ females and thus female sex hormones come into play in terms of effects on gene expression, is the fact that their probands were on average menopausal, and their controls on average pre-menopausal. Thus, these data are hard to rely upon, and general conclusions based on it are unwarranted.

That being said, better designed biomarker discovery and validation studies in depression, in females, and in other types of suicidality are areas that should be encouraged and vigorously pursued. Our group and others are actively working on this. It seems clear that there may be differences between bipolar disorder and depression, as shown here in Figure 1, where we reproduce in larger cohorts our previous findings of increased SAT1 and MARCKS, ${ }^{6}$ as well as independently reproduce the decreased SKA2 finding reported by Kaminsky and colleagues, ${ }^{8}$ more so in bipolar disorder than in depression.

An example of possible premature jumping to positive conclusions was also published recently. Redei et al. ${ }^{10}$ purport to have identified biomarkers for depression. These biomarkers were originally discovered in an animal model, of limited direct relevance or specificity to the human condition. There is a relative lack of transparency of why certain biomarkers were chosen and not others to carry forward in the current human study reported. Although there are some differences reported, there is no clear validation in independent human cohorts of a locked panel of markers, nor data on predictive ability for clinical course of the disorder in independent cohorts. In contrast, a panel of 10 biomarkers for mood state discovered by us in human studies of bipolar disorder subjects, locked and then validated by us in independent cohorts before publication, ${ }^{4}$ was recently replicated completely independently by another group and shown to track the course of response to cognitive-behavioral therapy in depression. ${ }^{11}$

Moving forward, it may be useful for our field to have a set of rules or postulates for what constitute good biomarkers, similar to the Koch postulates used in infectious diseases. First, working on the right problem. One has to be clear about the population used for discovery: for which well-defined phenotype, in what clinical diagnostic group, of what gender, the biomarker(s) have been discovered. Second, using the right approach. The experimental design used to discover biomarkers is crucial for signal detection. A within-subject design is more powerful than a case-case design with extremes of distribution, which in turn is more powerful than a case-control design, that would require a much higher number of subjects. Metabolomics might be more direct ${ }^{12}$ (albeit more limited) than proteomics, which in turn, if used in an unbiased discovery fashion, ${ }^{13}$ may be more powerful than gene expression, which in turn is more powerful than genetics, as thousands of singlenucleotide polymorphisms can converge in the regulation of expression of a gene. A convergent functional genomics ${ }^{3-6}$ or integrative approach to prioritize biomarkers based on multiple prior lines of evidence would ensure a fit to disease rather than a fit to cohort that may occur with machine learning approaches. Third, demonstrating reproducibility the right way. The validation of an a priori selected biomarker or locked panel of biomarkers in non-overlapping, completely independent cohorts with robust phenotypes is a must before making any believable claims. Fourth, the right use of the biomarkers. Showing prospectively that the biomarker(s) have predictive ability for future clinical course is necessary for the field to start adopting the biomarkers and to begin translating them into clinical practice, which should be a clear and present goal for all engaged in this type of research.

\section{CONFLICT OF INTEREST}

The authors declare no conflict of interest.

\section{ACKNOWLEDGMENTS}

We would particularly like to thank the veterans and other subjects who volunteered to participate in these studies, their families and their caregivers. Without their generous contribution, such work to advance the understanding of mental illness and help others would not be possible. We would like to thank Mike Yard, Alfarena Ballew and George Sandusky for help with collecting Coroner's Office cases; Elizabeth Belanger, Alison James, Sunita George, Holly Weber, Lauryn Myers, Robert Schweitzer and Dawn Graham for help with the human subjects testing and Terri Gelbart for excellent technical help on the microarray work. This work was supported by an NIH Directors' New Innovator Award (1DP2OD007363) and a VA Merit Award (2I01CX000139) to ABN.

\section{REFERENCES}

1 Park YY, Lee SS, Lim JY, Kim SC, Kim SB, Sohn BH et al. Comparison of prognostic genomic predictors in colorectal cancer. PLoS One 2013; 8: e60778.

2 Daniels SE, Beineke P, Rhees B, McPherson JA, Kraus WE, Thomas GS et al. Biological and Analytical Stability of a Peripheral Blood Gene Expression Score for Obstructive Coronary Artery Disease in the PREDICT and COMPASS Studies. J Cardiovasc Transl Res 2014; 7: 615-622.

3 Ayalew M, Le-Niculescu H, Levey DF, Jain N, Changala B, Patel SD et al. Convergent functional genomics of schizophrenia: from comprehensive understanding to genetic risk prediction. Mol Psychiatry 2012; 17: 887-905.

4 Le-Niculescu H, Kurian SM, Yehyawi N, Dike C, Patel SD, Edenberg HJ et al. Identifying blood biomarkers for mood disorders using convergent functional genomics. Mol Psychiatry 2009; 14: 156-174.

5 Kurian SM, Le-Niculescu H, Patel SD, Bertram D, Davis J, Dike C et al. Identification of blood biomarkers for psychosis using convergent functional genomics. $\mathrm{Mol}$ Psychiatry 2011; 16: 37-58.

6 Le-Niculescu H, Levey DF, Ayalew M, Palmer L, Gavrin LM, Jain N et al. Discovery and validation of blood biomarkers for suicidality. Mol Psychiatry 2013; 18: 1249-1264.

7 Punzi G, Ursini G, Shin JH, Kleinman JE, Hyde TM, Weinberger DR. Increased expression of MARCKS in post-mortem brain of violent suicide completers is related to transcription of a long, noncoding, antisense RNA. Mol Psychiatry 2014; 19: 1057-1059.

8 Guintivano J, Brown T, Newcomer A, Jones M, Cox O, Maher BS et al. Identification and replication of a combined epigenetic and genetic biomarker predicting suicide and suicidal behaviors. Am J Psychiatry advance online publication, 30 July 2014; doi:10.1176/appi.ajp.2014.14010008; e-pub ahead of print.

9 Mullins N, Hodgson K, Tansey KE, Perroud N, Maier W, Mors O et al. Investigation of blood mRNA biomarkers for suicidality in an independent sample. Transl Psychiatry 2014; 4: e474.

10 Redei EE, Andrus BM, Kwasny MJ, Seok J, Cai X, Ho J et al. Blood transcriptomic biomarkers in adult primary care patients with major depressive disorder undergoing cognitive behavioral therapy. Transl Psychiatry 2014; 4: e442.

11 Keri S, Szabo C, Kelemen O. Blood biomarkers of depression track clinical changes during cognitive-behavioral therapy. J Affect Dis 2014; 164: 118-122.

12 Kaddurah-Daouk R, Boyle SH, Matson W, Sharma S, Matson S, Zhu H et al. Pretreatment metabotype as a predictor of response to sertraline or placebo in depressed outpatients: a proof of concept. Transl Psychiatry 2011; 1. pii: e26.

13 Martins-de-Souza D, Maccarrone G, Ising M, Kloiber S, Lucae S, Holsboer F et al. Blood mononuclear cell proteome suggests integrin and ras signaling as critical pathways for antidepressant treatment response. Biol Psychiatry 2014; 76: e15-e17.

\footnotetext{
(c) (i) $\odot$

This work is licensed under a Creative Commons AttributionNonCommercial-NoDerivs 4.0 International License. The images or other third party material in this article are included in the article's Creative Commons license, unless indicated otherwise in the credit line; if the material is not included under the Creative Commons license, users will need to obtain permission from the license holder to reproduce the material. To view a copy of this license, visit http:// creativecommons.org/licenses/by-nc-nd/4.0/
} 